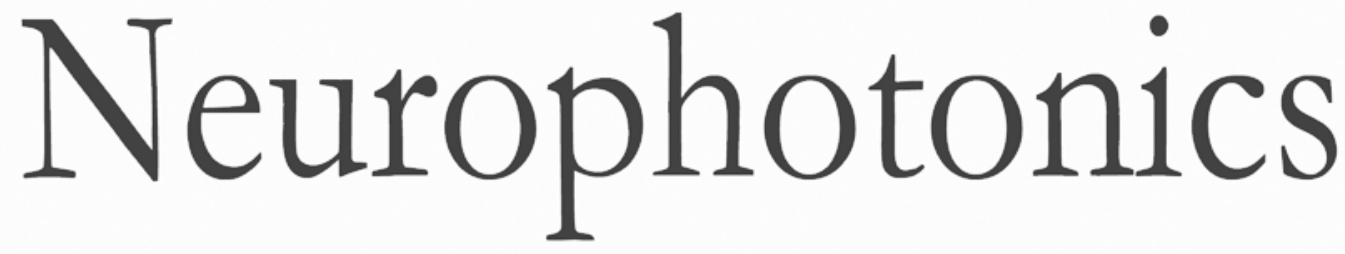

\title{
Nonresonant Raman spectroscopy of isolated human retina samples complying with laser safety regulations for in vivo measurements
}

\author{
Clara Stiebing \\ Iwan W. Schie \\ Florian Knorr \\ Michael Schmitt \\ Nanda Keijzer \\ Robert Kleemann \\ Izabella J. Jahn \\ Martin Jahn \\ Amanda J. Kiliaan \\ Laurin Ginner \\ Antonia Lichtenegger \\ Wolfgang Drexler \\ Rainer A. Leitgeb \\ Jürgen Popp
}




\title{
Nonresonant Raman spectroscopy of isolated human retina samples complying with laser safety regulations for in vivo measurements
}

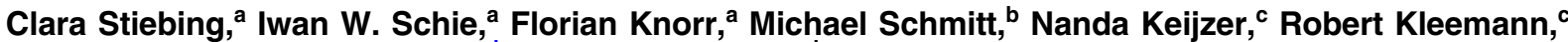 \\ Izabella J. Jahn, ${ }^{a}$ Martin Jahn, ${ }^{a, \dagger}$ Amanda J. Kiliaan, ${ }^{d}$ Laurin Ginner, ${ }^{e}$ Antonia Lichtenegger, ${ }^{e}$ \\ Wolfgang Drexler, ${ }^{\mathrm{e}}$ Rainer A. Leitgeb, ${ }^{\mathrm{e}}$ and Jürgen Popp ${ }^{\mathrm{a}, \mathrm{b}, *}$ \\ aLeibniz Institute of Photonic Technology, Albert-Einstein-Straße, Jena, Germany \\ ${ }^{\mathrm{b} F r i e d r i c h ~ S c h i l l e r ~ U n i v e r s i t y ~ J e n a, ~ I n s t i t u t e ~ o f ~ P h y s i c a l ~ C h e m i s t r y ~ a n d ~ A b b e ~ C e n t e r ~ o f ~ P h o t o n i c s, ~ H e l m h o l t z w e g, ~ J e n a, ~ G e r m a n y ~}$ \\ 'Netherlands Organisation for Applied Scientific Research, Department of Metabolic Health Research, Zernikedreef, Leiden, The Netherlands \\ ${ }^{\mathrm{d} R a d b o u d}$ University Medical Center, Institute for Brain, Cognition, and Behavior, Preclinical Imaging Center, Department of Anatomy Donders, \\ Nijmegen, The Netherlands \\ ${ }^{\mathrm{e}}$ Medical University of Vienna, Center for Medical Physics and Biomedical Engineering, Vienna, Austria
}

\begin{abstract}
Retinal diseases, such as age-related macular degeneration, are leading causes of vision impairment, increasing in incidence worldwide due to an aging society. If diagnosed early, most cases could be prevented. In contrast to standard ophthalmic diagnostic tools, Raman spectroscopy can provide a comprehensive overview of the biochemical composition of the retina in a label-free manner. A proof of concept study of the applicability of nonresonant Raman spectroscopy for retinal investigations is presented. Raman imaging provides valuable insights into the molecular composition of an isolated ex vivo human retina sample by probing the entire molecular fingerprint, i.e., the lipid, protein, carotenoid, and nucleic acid content. The results are compared to morphological information obtained by optical coherence tomography of the sample. The challenges of in vivo Raman studies due to laser safety limitations and predefined optical parameters given by the eye itself are explored. An in-house built setup simulating the optical pathway in the human eye was developed and used to demonstrate that even under laser safety regulations and the above-mentioned optical restrictions, Raman spectra of isolated ex vivo human retinas can be recorded. The results strongly support that in vivo studies using nonresonant Raman spectroscopy are feasible and that these studies provide comprehensive molecular information of the human retina. @ The Authors. Published by SPIE under a Creative Commons Attribution 4.0 Unported License. Distribution or reproduction of this work in whole or in part requires full attribution of the original publication, including its DOI. [DOI: 10.1117/1.NPh.6.4.041106] Keywords: Raman imaging; retinal imaging; optical coherence tomography; macular pigment; eye safety standard.
\end{abstract}

Paper 19035SSR received Apr. 10, 2019; accepted for publication Aug. 2, 2019; published online Sep. 3, 2019.

\section{Introduction}

Vision impairment is a frequently occurring condition in people over 50 years of age and experiences a steady increase in prevalence due to an aging society and population growth. ${ }^{1}$ However, the World Health Organization states that around $81 \%$ of occurrences could be avoided if diagnosed and treated early. ${ }^{2}$ Especially, retinal diseases such as diabetic retinopathy, glaucoma, and age-related macular degeneration (AMD) are leading causes of vision impairment in high-income countries. ${ }^{3}$ Hence, new approaches in ophthalmology are aiming at early diagnosis of retinal alterations. Typical diagnostic methods in the clinical environment range from direct fundus examination, i.e., the rear inner eye surface, by ophthalmoscopy to highresolution optical imaging, to fluorescence-based angiography and optical coherence tomography (OCT). Nowadays, OCT is routinely used in ophthalmology, due to its three-dimensional sectioning of the retina, allowing evaluation of subtle morphological changes in the underlying structure. OCT tomograms are recorded using laser interferometry by measuring the path

\footnotetext{
*Address all correspondence to Jürgen Popp, E-mail: juergen.popp@leibnizipht.de

†Present address: CiS Forschungsinstitut für Mikrosensorik GmbH, Erfurt,
} Germany length difference of backscattered light, due to changes in the refractive indices of different sample constituents. OCT angiography is a functional extension of OCT, which improves the diagnostic results, since not only the typical structural features in an OCT image are recorded but also blood vessels and blood perfusion can be visualized. ${ }^{4,5}$ Recently, advances have been made to speed up in vivo volumetric OCT imaging for ophthalmic surgery ${ }^{6}$ and to minimize aberration effects by applying adaptive optics during OCT measurements. ${ }^{7}$

Although the aforementioned techniques provide highquality structural information, they either lack chemical information or require labeling, e.g. for fluorescein angiography. In the last decade, there have been extensive developments in molecular spectroscopy for biomedical applications. A method that has gained wide attention in this context is Raman spectroscopy, which provides molecular specific information of biological samples without the need for administering external markers. Based on inelastic scattering of monochromatic light at molecular bonds, vibrations unique for particular molecular structures and functionalities are excited. All excited Raman active molecular vibrations in the laser focus compose the detected Raman spectra, which allows for differentiating macromolecules, such as protein, nucleic acids, and lipids based on their intrinsic molecular signature. ${ }^{8,9}$ The combination of Raman spectroscopy with confocal microscopic imaging is well-suited 
for tissue and cellular imaging since a spatial resolution down to the diffraction limit can be achieved. Raman spectroscopy has been widely used for a range of biomedical applications for the detection and differentiation of cancer, infectious diseases, or cell-drug interactions. ${ }^{10-12}$ Although previous studies have indicated that Raman spectroscopy has promising applications for ophthalmology, ${ }^{13,14}$ only a few studies have used Raman spectroscopy under nonresonant conditions with excitation wavelength at $633 \mathrm{~nm}$ or above, i.e., outside an electronic absorption of most biological materials. For example, different biomarkers have been identified for monitoring retinal inflammation, ${ }^{15}$ and retinal changes occurring in glaucoma were investigated. ${ }^{16}$ The combination of morphologically sensitive methods, such as OCT with Raman spectroscopy has been successfully demonstrated on various biological tissues ${ }^{17-20}$ and could have profound implications for ophthalmology. Initial combinations of those modalities have readily been performed on ex vivo porcine and human retina tissue..$^{21}$ The study showed that the key challenge for using nonresonant Raman spectroscopy in ophthalmology is the restriction of low excitation laser powers in accordance with safety regulations, leading to low signal intensities. Therefore, most studies focus on resonance Raman spectroscopy (RRS) using an excitation wavelength in the visible range below $633 \mathrm{~nm}$. Here the Raman excitation wavelength is close to or coinciding with an excited electronic state of molecules in the sample, leading to an enhancement of the weak Raman signal. This significantly boosts the low intensity of the Raman signal and allows acquisition of Raman spectra with laser excitation intensities complying with common safety regulations. However, RRS leads only to an enhancement of modes, which are coupled to electronic chromophores, i.e., mainly probes pigments. In the case of the retina, these are carotenoids such as lutein and zeaxanthin. ${ }^{22,23}$ Due to their antioxidant nature, they are considered to play an important role for protecting the human eye against visual loss from AMD. ${ }^{24}$ First in vivo RRS studies evaluated the macular carotenoid levels of these pigments based on individuals of different ages, ${ }^{25}$ as well as AMD patients and healthy volunteers. ${ }^{26}$ RRS was also used to investigate the delivery of lutein and zeaxanthin to the human retina by trans-scleral iontophoresis in an ex vivo study ${ }^{27}$ and orally in an in vivo clinical trial study. ${ }^{28}$ However, conflicting results have been reported on the diagnostic value of RRS for the determination of macular pigment levels when compared to the more established heterochromatic flicker photometry, ${ }^{29,30}$ leading to the conclusion that the two methods cannot be simply correlated and further research is needed. A major drawback of RRS turns out to be that the enhanced Raman bands dominate the spectral profile, significantly reducing information of many biological substances of interest, which do not contain chromophores. Due to these shortcomings, it would be highly desirable to use nonresonant Raman spectroscopy, because in contrast to RSS, it provides the entire molecular fingerprint, i.e., the lipid, protein, carbohydrates, carotenoids, and nucleic acid content of a sample. Thereby, a higher specificity and a greater diagnostic value would be achieved. Although it has been already demonstrated that nonresonant Raman spectroscopy is a promising method for studying the biochemical composition and disease related changes of ex vivo eye samples, ${ }^{31-33}$ in vivo studies of the human retina have not yet been performed. The transition of Raman spectroscopy into the clinics is challenging especially for ophthalmology. Due to the use of a laser as an excitation source safety regulations have to be followed, e.g., the
European DIN EN ISO 15004-2 (2007) and the US American ANSI Z136.1 norms. What should not be neglected is that the eye itself is an optical system with predefined parameters, which impairs the excitation and detection path for in vivo Raman spectroscopy of the retina.

In this contribution, we demonstrate to the best of our knowledge for the first time the applicability of nonresonant Raman spectroscopy on isolated ex vivo human retina complying with international laser safety regulations for the permissible excitation power. Furthermore, we are presenting an optical setup that mimics the optical parameters of the human eye and show in a proof of concept study that it is indeed possible to acquire nonresonant Raman spectra of retinas, providing access to the macromolecular content, such as proteins, lipids, and carotenoids. By performing OCT on the same samples, it is also possible to relate the molecular signatures to morphological information.

\section{Materials and Methods}

\subsection{Sample Preparation}

Measurements were performed on human retina samples acquired via the body donor program at the Department of Anatomy of the Radboud University Medical Centre (Radboudumc), Nijmegen, The Netherlands. All body donors in this program signed a written informed consent during their lifetime permitting the use of their body and parts for science and teaching. The samples were transferred from Radboudumc to the Netherlands Organisation for Applied Scientific Research (TNO), Department of Metabolic Health Research, Leiden, The Netherlands. The isolation was performed under the guidelines from the Department of Anatomy of the Radboudumc and approved by the Medical Ethics Review Committee region Arnhem-Nijmegen (Commissie Mensgebonden Onderzoek CMO region Arnhem-Nijmegen file No. 2017-3941). Anonymized medical records were provided with the transfer of the samples.

Eyes were donated by two female and one male person, all in the age range between 86 and 91 years of age. Herein, the presented measurements were performed on a retina donated by an 89-year old male person diagnosed with Alzheimer's disease. Measurements of the two female donors were excluded due to disintegrated retinal layers most likely due to a relatively long period of time $(20 \mathrm{~h})$ between death and enucleation. The presented donor eyes were enucleated within $13 \mathrm{~h}$ after death at the Department of Anatomy of the Radboudumc and stored at $-80^{\circ} \mathrm{C}$. The samples were shipped to TNO Leiden and stored at $-80^{\circ} \mathrm{C}$ until dissection. Eyes were carefully thawed in $4^{\circ} \mathrm{C}$ cold $1 \times$ PBS for the duration of $1 \mathrm{~h}$ and afterward transferred onto a gauze soaked in cold $1 \times$ PBS in a Petri dish. The cornea was removed by first making an incision at the limbus into the anterior chamber. Scissors were then inserted into the anterior chamber and circumferential cuts were made in the cornea, just anterior to the limbus, to remove the lens and iris. The eye was opened up by making four equally spaced cuts from the ciliary body toward the optic nerve head. The vitreous was removed and the retina was carefully lifted with forceps and placed on $\mathrm{CaF}_{2}$ slides, where the retina was immediately frozen with dry ice. The whole isolation procedure was performed within about $10 \mathrm{~min}$. Raman and OCT measurements were done on dissected en face retina of the posterior segment of the frozen eye. 


\subsection{Raman Spectral Acquisition}

To acquire Raman images of isolated en face human retina samples, a conventional confocal Raman microscope alpha300 R (WITec, Ulm, Germany) equipped with an Olympus $10 \times$ air objective [numerical aperture (NA) of 0.25 ] and a continuous wave diode laser providing an excitation wavelength of $785 \mathrm{~nm}$ (Toptica Photonics, Gräfelingen, Germany) was used. The power was adjusted to $20 \mathrm{~mW}$ at the sample plane. An integration time of $5 \mathrm{~s}$ and a step size of $75 \mu \mathrm{m}$ were chosen to record Raman images, after the retina sample dried under ambient conditions. With a $300 \mathrm{~g} / \mathrm{mm}$ grating, the complete wavenumber region from 100 to $3300 \mathrm{~cm}^{-1}$ was recorded with a spectral resolution of around $6 \mathrm{~cm}^{-1}$.

To advance toward in vivo-like measurement conditions, an in-house built Raman setup was designed and realized following the optical parameters of the eye. The setup was connected to a Princeton Instruments (PI, Trenton, New Jersey) LS-785 high-throughput lens spectrograph including a continuous wave diode laser FER785-MM from PI and equipped with a PIXIS back-illuminated deep depletion charge-coupled device (PIXIS100, PI) camera cooled to $213 \mathrm{~K}$. The laser light is collimated by a plano-convex lens and passes a laser clean-up filter for blocking the in-fiber generated Raman signal. A 45-deg dichroic beamsplitter directs the laser light onto an achromatic lens, which focuses the excitation light onto the sample. The same objective lens is used for the collection of the generated Raman signal. After passing a single-notch filter, used to suppress the Rayleigh scattered light, the collected light is coupled into the detection fiber. The optical parameters of the fibers and lenses have been adapted to the optical components of the housed PI system and in order to achieve a spot size of $100 \mu \mathrm{m}$ on the sample surface while having the NA of 0.147 , corresponding to NA observed in the human eye. A neutral density filter was installed into the excitation path behind the optical fiber input, to reduce the laser power to $1 \mathrm{~mW}$ at the sample plane to comply with the laser power limitation for eye safety.

\subsection{Raman Data Analysis}

All data processing was performed in $\mathrm{R}^{34}$ using as main packages hyperSpec ${ }^{35}$ and cbmodels. ${ }^{36}$ All spectra were cut to a wavenumber region between 400 and $3100 \mathrm{~cm}^{-1}$ and the wavenumber axes calibrated, using paracetamol as a reference. An extended multiplicative scatter correction (EMSC) algorithm implementing four background component spectra and one pure component spectrum was used as a baseline correction with prior interpolation to assimilate the wavenumber axes. The background components were an offset, a linear function, and two sets of fluorescence signals. All spectra were cleared from cosmic spikes by an in-house written algorithm. ${ }^{37}$ Raman images recorded with the commercial WITec system were analyzed by a hierarchical cluster analysis (HCA) algorithm ${ }^{38}$ using a Pearson distance calculation and Ward clustering algorithm providing cluster centroids that represent weighted average spectra for each cluster. ${ }^{39}$ A quality control was performed to remove spectra with no relevant Raman information, i.e. too strong fluorescence background or positioned outside the sample. Raman spectra recorded with the in-house built setup were either plotted as raw data without any processing except wavenumber calibration or smoothed and baseline corrected as described above. For graphical representation, the spectra were normalized as indicated.

\subsection{OCT Imaging}

The OCT volume was recorded with a custom-built swept source OCT system with a central wavelength of $1050 \mathrm{~nm}$ and a bandwidth of $110 \mathrm{~nm}$, resulting in an axial resolution of $4.5 \mu \mathrm{m}$ in air. The implemented swept source AXSUN A1300467 provides an operating speed of $200 \mathrm{kHz}$ and a measured sensitivity of $94 \mathrm{~dB}$. For the image acquisition, a dual-balanced photodetector Exalos 1040392 with a digitizer Alazartech ATS9350 card is used. The two-dimensional scanning is performed with Cambridge technology scanners, offering a field of view of max. $40 \mathrm{deg}$. The illumination power was set to $1.2 \mathrm{~mW}$, which is below the permissible exposure limit of the laser safety standards for in vivo ophthalmic imaging. The beam size is $1.4 \mathrm{~mm}$ and the last lens used a $30-\mathrm{mm}$ achromatic lens (Thorlabs AC254-030-B), leading to a lateral resolution of max. $24 \mu \mathrm{m}$. Volumes are patches consisting of $400 \times 800 \times 400$ pixel $(x, y$, and $z$ ). Image recording, reconstruction, and processing were performed using LabView and ImageJ.

\section{Results}

To test whether reliable nonresonant Raman spectra can be acquired from human retina samples confocal Raman imaging was performed on a conventional Raman microscope with an excitation wavelength of $785 \mathrm{~nm}$ and $20 \mathrm{~mW}$ laser power on the sample plane. Representative bright-field and Raman images of an isolated en face retina are shown in Figs. 1(a) and 1(b), respectively. No significant structural features can be observed in the bright-field image [Fig. 1(a)] for hydrated retina. Due to long acquisition times, Raman imaging was performed after the retina dried under ambient conditions. The Raman spectra of the entire dataset in Fig. 1(b) are plotted as a mean spectrum with standard deviation in Fig. 1(c). It is obvious that recording Raman spectra is challenging due to the simultaneous excitation of endogenic fluorophores within the retina or the retinal pigment epithelium, which is located in close proximity beneath the retina. Autofluorescence leads to an intense, broad background in the spectra, causing a low signal-to-noise ratio for Raman bands. Although the background is high, small contributions of typical protein and lipid bands at $1442 \mathrm{~cm}^{-1}\left(\mathrm{CH}_{2}\right.$ bending) and $1655 \mathrm{~cm}^{-1}(\mathrm{C}=\mathrm{C}$ stretching $)$ as well as the $\mathrm{CH}$ stretch region between 2800 and $3050 \mathrm{~cm}^{-1}$ can be observed.

The high background was successfully removed by the EMSC algorithm, using fluorescence signals as background components (for details see Sec. 2). Only 264 spectra out of the initial 4020 spectra were discarded during quality control, including 159 spectra due to such strong fluorescence background that no Raman information was retrievable. After preprocessing, HCA was performed, creating a cluster image with the corresponding cluster centroids (Fig. 2). In total, five clusters were used to define the Raman image displayed in Fig. 2(a) as an overlay on top of the bright-field image.

The cluster centroids [Fig. 2(b)] are dominated by the aforementioned Raman bands of lipids and proteins at 1442 and $1655 \mathrm{~cm}^{-1}$. The band at $1002 \mathrm{~cm}^{-1}$ can be assigned to ring breathing modes of phenylalanine. Difference can be seen in the lower signal-to-noise ratio of cluster 2 as compared to cluster 1 due to high-fluorescence contributions, which was successfully removed with the baseline algorithm but is clearly visible in the raw data. Although clusters 1 to 4 mainly contain features of proteins, considerable differences can be seen in bands associated to nucleic acids at 751,1344 , and $1581 \mathrm{~cm}^{-1}$ present in the 

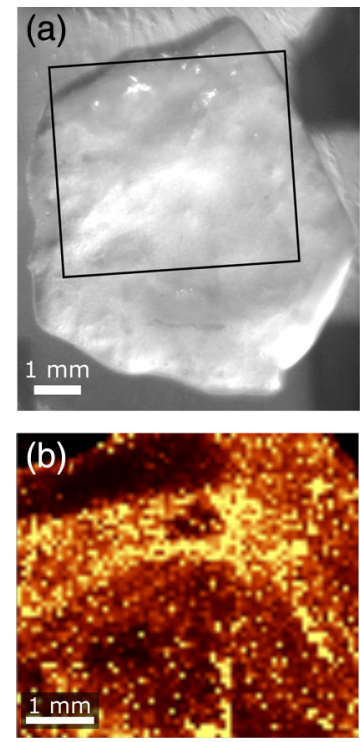

(c)

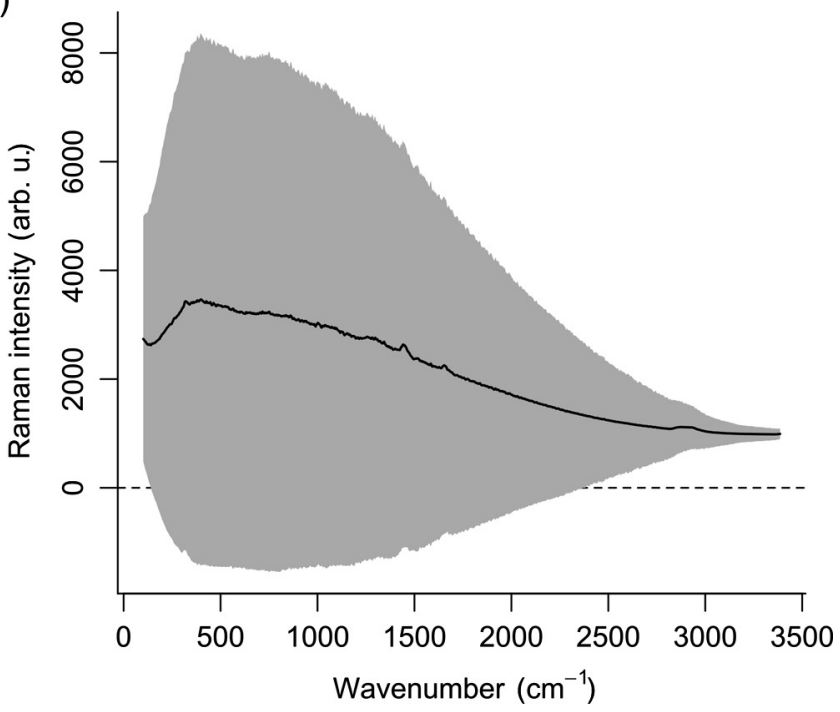

Fig. 1 (a) Representative bright-field image of isolated human retina before drying with the Raman measurement area indicated with a black box. (b) Raman intensity map using the wavenumber region from 2800 to $3050 \mathrm{~cm}^{-1}$. (c) Raw mean spectrum including standard deviation of the entire Raman dataset.
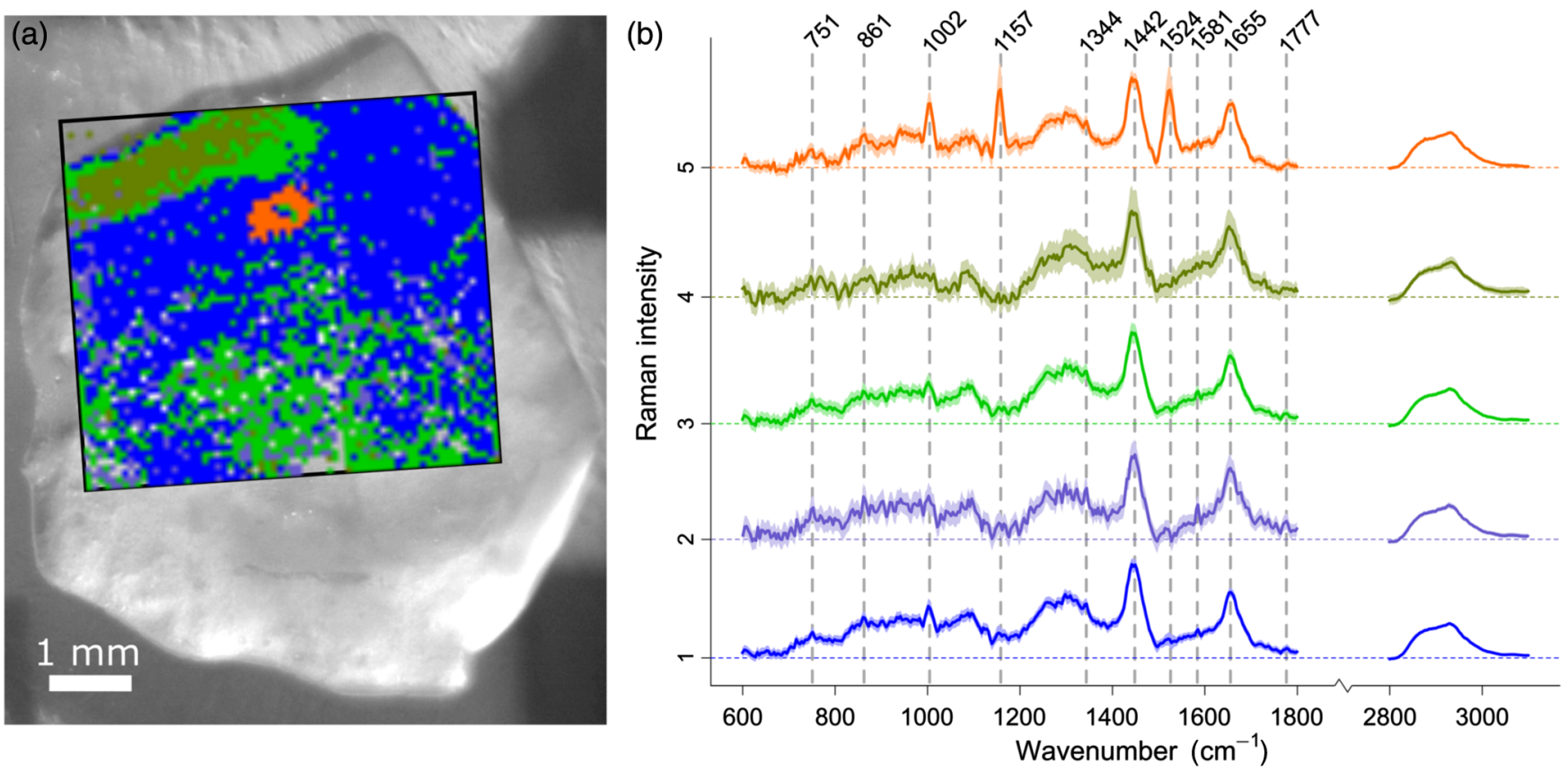

Fig. 2 (a) Bright-field image with overlaid HCA cluster Raman image of a human retina sample and (b) the corresponding cluster centroids with standard deviation. For better visualization, the spectra were area-normalized to the $\mathrm{CH}$ stretching region between 2800 and $3050 \mathrm{~cm}^{-1}$. The cluster image (a) and spectra (b) have corresponding color coding.

cluster 1 to 3 . The bands are most pronounced in cluster 2, indicating higher nucleic acid content. The bands at 861 and $1777 \mathrm{~cm}^{-1}$ in clusters 1 and 2 are indicative of a higher esterified lipid and phospholipid content. Contrarily, cluster 5 shows really intense bands at 1157 and $1524 \mathrm{~cm}^{-1}$, which can be assigned to carotenoids, most likely lutein or zeaxanthin as being the most abundant carotenoids in the retina. A precise assignment is not straightforward due to their similar molecular structure. The image reveals that the orange cluster is concentrated in only a small area with a diameter of $\sim 0.6 \mathrm{~mm}$. The size and elevated carotenoid content strongly suggests that this area is representing the fovea as part of the macula, a highly pigmented area near the center of the retina. After the drying process of the sample, the described area revealed yellowish discoloration, which was not visible in the moist state.

Next to confocal Raman imaging, a 3-D OCT volume was recorded to demonstrate the increased diagnostic value of combining OCT as a structural method with Raman providing additional molecular information. The custom-designed swept source OCT system was specifically designed for ophthalmic 

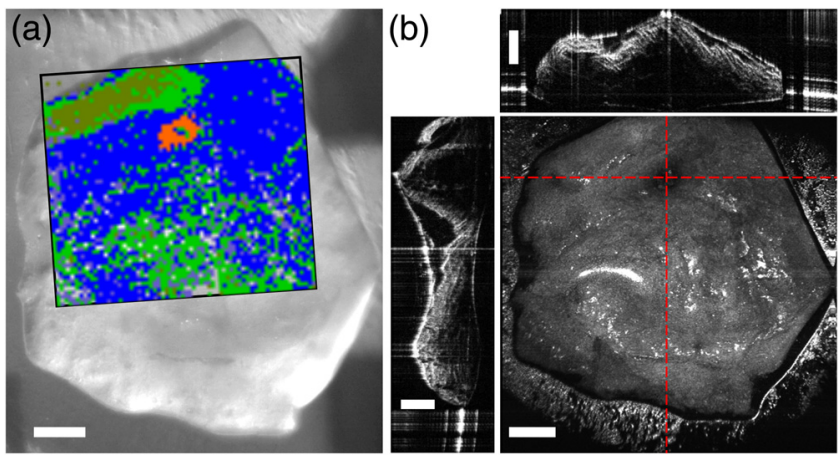

Fig. 3 (a) Bright-field image with overlaid Raman cluster image displayed in Fig. 2(a) and orthogonal cross sections through the recorded OCT volume together with the maximum intensity projection en face OCT image in (b) of a human retina sample. The red lines in the en face image display the position of the depth B scans on the top and left. White bars represent scale bars of $1 \mathrm{~mm}$.

diagnostics. ${ }^{40}$ Figure 3 shows the correlation between these two techniques. Although the bright-field image does not show any structural differences [Fig. 1(a)], the aforementioned spot [orange cluster in Fig. 2(a)] appears in the OCT projection view as a darker area, which might be due to the lower retinal tissue content indicative for the foveal region. However, only by adding the biochemical information of Raman spectroscopy, the molecular identification of a high carotenoid content is possible. The OCT depth B scans reveal that the typical layered structure of retinas, apart from spurious nerve fiber structures, was disturbed, possibly due to disintegration processes starting upon death.

For in vivo ophthalmic diagnostics of the retina, Raman imaging is not feasible. Due to the weak nonresonant Raman scattering process, the acquisition of an image takes up to several hours, e.g., the total acquisition of the retinal Raman image shown in Fig. 1(b) took about 6 h. Furthermore, certain criteria have to be considered for in vivo measurements in the human eye. As mentioned in Sec. 1, laser safety regulations have to be followed to ensure the safety of the patient. Considering the relevant laser safety standards, the strongest limitation is set by norm DIN EN ISO 15004-2 (2007), which defines the maximum exposure limit for ophthalmic instruments directly at the retina. A spectral weighting factor needs to be considered to account for absorption properties of the retina. In total, the effective exposure limit results in $1.4 \mathrm{~mW}$ excitation power for an excitation wavelength of $785 \mathrm{~nm}$ and a spot diameter of $100 \mu \mathrm{m}$. Although for such a configuration, the permissible exposure time of up to $5 \mathrm{~min}$ is rather long, the exposure time for in vivo measurements in the eye needs to be restricted to a fraction of a minute to minimize critical motion distortions and loss of fixation. Another factor to consider is that the eye itself is an optical system with predefined parameters. The axial length between cornea and retina is ca. $24 \mathrm{~mm}$ with a refractive power of the nonaccommodated eye of about $60 \mathrm{~m}^{-1}$. This leads to an effective focal length of $22 \mathrm{~mm}$, considering the refractive index of the ocular media to be about 1.34. To achieve the largest physical aperture, the pupil should be fully dilated, which can be induced, e.g. by tropicamide, and is routinely performed during eye examinations. The maximum pupil size then results in ca. $7 \mathrm{~mm}$. With a collimated beam entering the pupil, a lateral spot size of $100 \mu \mathrm{m}$ is considered to be present at the retina. This value results in a variable NA, which needs to be matched with the detection path and the spectrometer. The highest collection NA for the Raman photons provided by the geometry of the eye is 0.147 . To simulate these optical parameters in the laboratory, an in-house built setup was designed, ensuring the aforementioned parameters. Figure 4 shows the designed probe and implemented device. It is based on fiber delivery of the excitation light and fiber collection for the Raman photons. The optical parameters of the fibers and lenses where adapted in order to achieve a spot size of $100 \mu \mathrm{m}$ on the sample surface and matching the $f \#$ of the spectroscopy system while ensuring a collection NA of 0.147 for the objective lens. The specifications are provided in Fig. 4 and detailed in Sec. 2. Overall, the key differences of the in-house built setup compared to the commercial one used for Raman imaging (Fig. 1) are the reduced confocality and increased focal depth, the low collection NA of 0.147 , and the relatively large detection spot size of $100 \mu \mathrm{m}$ on the sample.

The in-house built setup was used to investigate the retina sample under in vivo-like conditions. To comply with the laser safety regulations, the laser power in the sample plane was set below the limit to $1 \mathrm{~mW}$. Figure 5(a) depicts the bright-field

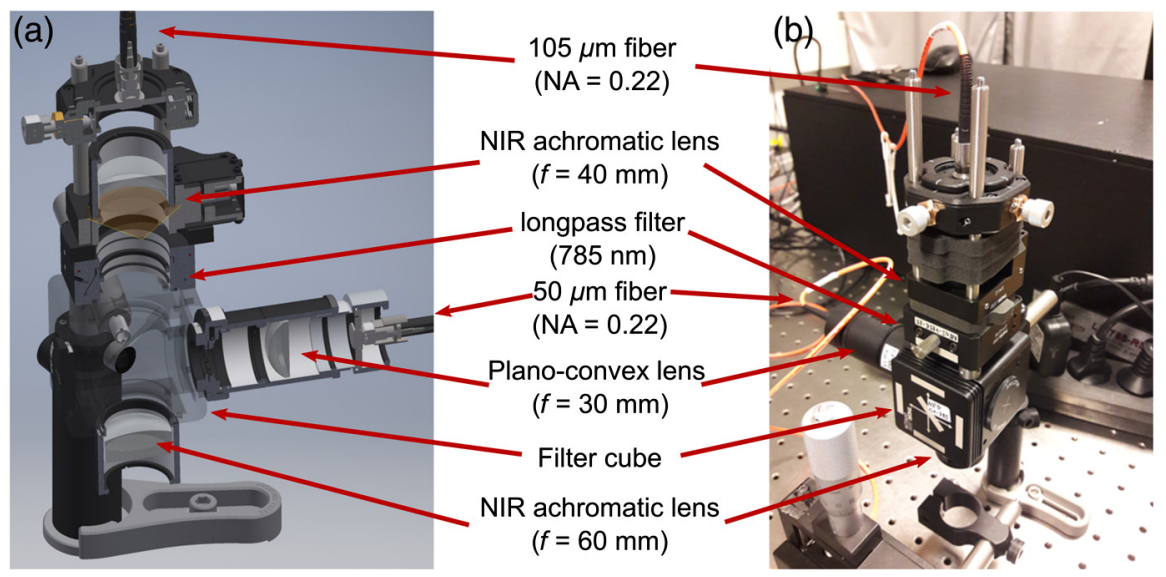

Fig. 4 (a) Mechanical layout and (b) picture of the in-house built Raman setup to simulate the optical pathway in the eye. The setup allows for simulating the in vivo parameters in the eye and to minimize the laser power to $1 \mathrm{~mW}$ to comply with safety regulations. 

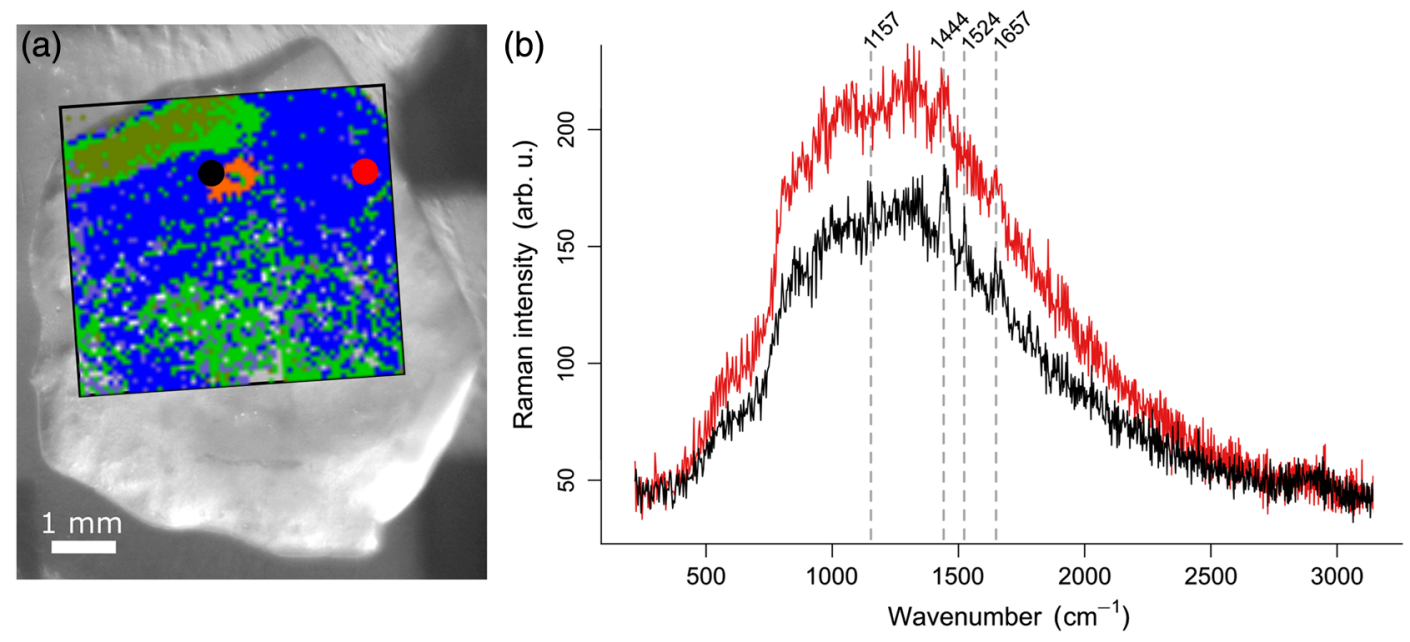

Fig. 5 (a) Bright-field image with overlaid Raman cluster image of a human retina sample with two Raman measurement positions marked in black and red. (b) Unprocessed mean Raman spectra recorded with the in-house built setup at the two positions marked by the black and red spot in (a) with the corresponding color coding.

image with the overlaid Raman cluster image of Fig. 2 with two spots in black and red marking the Raman measurement positions with the in-house built setup. These two spots were chosen in order to obtain spectra from an area with (black) and without (red) carotenoid contributions based on the previous Raman imaging results (see Fig. 2). The corresponding Raman spectra are shown in Fig. 5(b) as unprocessed means of three accumulations each recorded with $10 \mathrm{~s}$ integration time. Despite three accumulations, both mean spectra have a high noise level compared to the Raman signals, but still typical bands of retinal tissue at 1444 and $1657 \mathrm{~cm}^{-1}$ can readily be identified from the uncorrected spectra. Furthermore, the distinction between the two spectra based on the carotenoid bands at 1157 and $1524 \mathrm{~cm}^{-1}$ is still possible. The bands only appear in the black spectrum corresponding to the orange cluster of the confocal Raman image.

The two mean spectra shown in Fig. 5(b) were both subjected to the same baseline correction as described above with an additional smoothing algorithm to further reduce the strong noise contributions. This preprocessing achieved an increase in spectral quality, and the main Raman bands are clearly retrieved [Figs. 6(a) and 6(b)]. Figure 6 provides a direct comparison of the two processed spectra (a) and (b) with spectra obtained under optimal conditions at the confocal imaging setup (c) and (d). To compare the obtained Raman information, the measurement spots used for the in-house built system were transferred to the Raman image and four neighboring spectra from the indicated region were extracted and averaged, covering an area of $5700 \mu \mathrm{m}^{2}$, to match the area covered by the excitation spot size of $100 \mu \mathrm{m}$ of the in-house built system. The spectra were processed with the same baseline and smoothing algorithm and normalized for a better visualization. The Raman spectra recorded under in vivo-like conditions using the in-house built system agree well with the results obtained under the optimal conditions using the confocal Raman imaging system. Certain deviations can be seen, which are likely due to the reduced confocality of the in-house optical setup, probing also other molecular contributions of the retina.
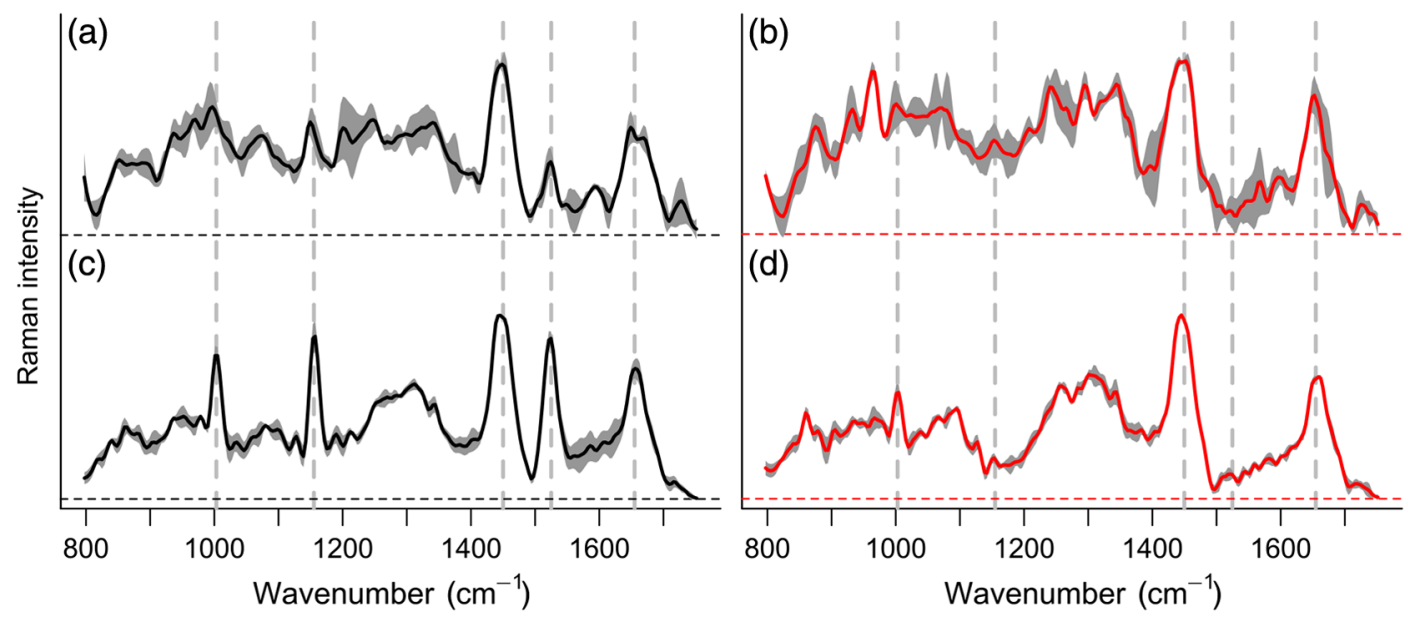

Fig. 6 Comparison of mean Raman spectra including standard deviation at the same position of the human retina sample [indicated in Fig. 5(a)] with the (a) and (b) in-house built system, and (c) and (d) confocal imaging system. The spectra were baseline corrected, smoothed, and $\mathrm{min} / \mathrm{max}$-normalized. 


\section{Discussion}

Using a Raman excitation wavelength of $785 \mathrm{~nm}$, reliable nonresonant Raman spectra of human retinal tissue components were recorded. It was possible to remove the high-fluorescence background properly to retain high-quality Raman spectra with defined Raman bands. The excitation wavelength of $785 \mathrm{~nm}$ offers the best compromise between fluorescence suppression, due to an excitation away from an electronic transition, and acceptable signal levels although the intensities decrease with $\lambda^{-4}$. In the presented Raman image [see Fig. 2(a)], a homogeneous distribution of proteins was found as well as the presence of esterified lipids and phospholipids. Since the commercial Raman system has a high confocality, mostly information of the top layers of the retina was collected. Due to the presence of nucleic acid bands, contributions of the inner nuclear layer are likely. Next to lipid and protein content, it was possible to also retrieve information of carotenoids under nonresonant excitation conditions. In many studies, information on the latter is the only information retrieved since the majority of Raman studies on retinas were performed under electronic resonant conditions in a lower wavelength region, i.e., using RRS. The nonresonant $785 \mathrm{~nm}$ excitation approach presented herein is uniquely qualified to detect concentration differences in organic material and hence can discern the carotenoid-rich fovea from the rest of the retina as the bands are easily seen in the corresponding orange cluster but not in the others. With RRS, this is, however, more difficult due to the specific and high enhancement of carotenoid bands even in areas with low carotenoid concentrations. Nonresonant Raman spectroscopy can, therefore, provide a much more comprehensive overview of critical components of human retinas and their biochemical composition, e.g., compositional changes in the fovea, resulting in a greater diagnostic value.

To the best of our knowledge, this is the first time that nonresonant Raman imaging was used to study human retina samples providing information about their molecular composition, i.e., lipids, proteins, and nucleic acids, and revealing highly localized macromolecular distribution of carotenoids. Raman imaging is not applicable for in vivo studies, due to the intrinsically weak process leading to long acquisition times to record an entire retina Raman image. A multimodal platform combining Raman spectroscopy with OCT can provide a multitude of advantages because OCT imaging can rapidly provide a structural overview and define interesting locations for a specific molecular profiling using nonresonant Raman spectroscopy, leading to a significant decrease of the acquisition time. To demonstrate the powerful combination of both modalities, OCT volumes on the same retina samples were recorded. The OCT projection view agrees well with the en face Raman image and further indicates that the localized carotenoid contribution seen in the Raman image represents the foveal region. However, the OCT depth B scans did not show the typical layered structure of retinas. This is most likely due to tissue disintegration during the relatively long period between occurrence of death and the time point at which the eye tissue was frozen. Disintegration processes in the eye start right after death, which causes the retina to spontaneously detach from the eyeball during dissection and thereby causes disturbances of its layered structure.

To validate the applicability of nonresonant Raman spectroscopy for the anticipated in vivo studies, measurement conditions were adjusted based on the predefined conditions faced when measuring in the eye. The strongest restrictions were set by the low collection NA of 0.147 and the low laser power of $1 \mathrm{~mW}$. Still, Raman information of the retinal tissue can be recorded and the differentiation of the foveal region was possible. An averaging of three spectra each with $10 \mathrm{~s}$ integration time was chosen to keep motion distortions during in vivo studies to a minimum while still recording good signal intensities. To improve the comfort for patients, an averaging of multiple short measurements, rather than one extended integration, is favorable. The results from the in-house setup were compared with the conventional, confocal microscopy setup, providing comparable results. These findings clearly point out that even under significantly restricted conditions Raman information of human retina can be retrieved.

\section{Conclusion}

In contrast to previous studies, ours was able to obtain a comprehensive overview of the macromolecular information of human retinas. The nonresonant Raman spectroscopy approach identified not only carotenoids but also other molecular species (e.g., proteins, lipids, and nucleic acids) that are valuable for ophthalmic diagnostics. By developing a Raman setup, which mimics the optical parameters for the Raman signal collection of a human eye, it was also possible to acquire Raman spectra complying with international laser standards for permissible laser exposure to the human eye. The presented results strongly support that in vivo studies using nonresonant Raman spectroscopy for a molecular characterization of the human retina will be achievable. The findings also outline great potential for a combined OCT/Raman morpho-chemical characterization of human retina in new ophthalmological studies of age-related degenerative diseases. In ongoing studies conducted by the authors, fast OCT imaging is used to identify suspicious regions for subsequent in-depth molecular characterization using Raman spectroscopy. This multimodal combination will significantly extend currently available analytical methods in ophthalmology and allow the determination of novel biomarkers for retinal diagnostics.

\section{Disclosures}

The authors declare that they have no conflicts of interest.

\section{Acknowledgments}

The authors acknowledge financial support from the European Union's Horizon 2020 Research and Innovation Program (MOON H2020-ICT-2016-1, No. 732969).

\section{References}

1. R. R. A. Bourne et al., "Magnitude, temporal trends, and projections of the global prevalence of blindness and distance and near vision impairment: a systematic review and meta-analysis," Lancet Global Health 5(9), e888-e897 (2017).

2. World Health Organization (WHO), Eye Care (2019), https://www.who .int/pbd/blindness/WorldSightDay17Infographic.pdf.

3. World Health Organization (WHO), "Universal eye health: a global action plan 2014-2019," https://www.who.int/blindness/AP2014_19_ English.pdf (2013).

4. R. A. Leitgeb et al., "Doppler optical coherence tomography," Prog. Retinal Eye Res. 41, 26-43 (2014).

5. J. Chua et al., "Future clinical applicability of optical coherence tomography angiography," Clin. Exp. Optom. 102(3), 260-269 (2019).

6. O. M. Carrasco-Zevallos et al., "Constant linear velocity spiral scanning for near video rate 4D OCT ophthalmic and surgical imaging with isotropic transverse sampling," Biomed. Opt. Express 9(10), 5052-5070 (2018). 
7. M. Salas et al., "Visualization of micro-capillaries using optical coherence tomography angiography with and without adaptive optics," Biomed. Opt. Express 8(1), 207-222 (2017).

8. Y. Shen, F. Hu, and W. Min, "Raman imaging of small biomolecules," Annu. Rev. Biophys. 48, 347-369 (2019).

9. C. Krafft et al., "Label-free molecular imaging of biological cells and tissues by linear and nonlinear Raman spectroscopic approaches," Angew. Chem. Int. Ed. Engl. 56(16), 4392-4430 (2017).

10. K. Eberhardt et al., "Advantages and limitations of Raman spectroscopy for molecular diagnostics: an update," Expert Rev. Mol. Diagn. 15(6), 773-787 (2015).

11. I. Pence and A. Mahadevan-Jansen, "Clinical instrumentation and applications of Raman spectroscopy," Chem. Soc. Rev. 45(7), 1958-1979 (2016).

12. C. Krafft et al., "Developments in spontaneous and coherent Raman scattering microscopic imaging for biomedical applications," Chem. Soc. Rev. 45(7), 1819-1849 (2016).

13. R. J. Erckens et al., "Raman spectroscopy in ophthalmology: from experimental tool to applications in vivo," Lasers Med. Sci. 16(4), 236-252 (2001).

14. J. V. Glenn et al., "Confocal Raman microscopy can quantify advanced glycation end product (AGE) modifications in Bruch's membrane leading to accurate, nondestructive prediction of ocular aging," FASEB $J$. 21(13), 3542-3552 (2007).

15. M. Marro et al., "Dynamic molecular monitoring of retina inflammation by in vivo Raman spectroscopy coupled with multivariate analysis," J. Biophotonics 7(9), 724-734 (2014).

16. Q. Wang et al., "Exploring Raman spectroscopy for the evaluation of glaucomatous retinal changes," J. Biomed. Opt. 16(10), 107006 (2011).

17. M. Wollweber and B. Roth, "Raman sensing and its multimodal combination with optoacoustics and OCT for applications in the life sciences," Sensors 19(10), 2387 (2019).

18. J. R. Maher et al., "Co-localized confocal Raman spectroscopy and optical coherence tomography (CRS-OCT) for depth-resolved analyte detection in tissue," Biomed. Opt. Express 6(6), 2022-2035 (2015).

19. J. Wang et al., "Characterizing biochemical and morphological variations of clinically relevant anatomical locations of oral tissue in vivo with hybrid Raman spectroscopy and optical coherence tomography technique," J. Biophotonics 11(3), e201700113 (2018).

20. C. A. Patil et al., "Integrated system for combined Raman spectroscopyspectral domain optical coherence tomography," J. Biomed. Opt. 16(1), 011007 (2011).

21. J. W. Evans et al., "Optical coherence tomography and Raman spectroscopy of the ex-vivo retina," J. Biophotonics 2(6-7), 398-406 (2009).

22. W. Gellermann et al., "Raman imaging of human macular pigments," Opt. Lett. 27(10), 833-835 (2002).

23. P. S. Bernstein et al., "Resonance Raman measurement of macular carotenoids in the living human eye," Arch. Biochem. Biophys. 430(2), 163-169 (2004).

24. B. Li, P. Vachali, and P. S. Bernstein, "Human ocular carotenoid-binding proteins," Photochem. Photobiol. Sci. 9(11), 1418-1425 (2010).

25. W. Gellermann et al., "In vivo resonant Raman measurement of macular carotenoid pigments in the young and the aging human retina," J. Opt. Soc. Am. A 19(6), 1172-1186 (2002).

26. P. S. Bernstein et al., "Resonance Raman measurement of macular carotenoids in normal subjects and in age-related macular degeneration patients," Ophthalmology 109(10), 1780-1787 (2002).

27. M. Lombardo et al., "Assessment of trans-scleral iontophoresis delivery of lutein to the human retina," J. Biophotonics 11(3), e201700095 (2018).

28. S. Beatty et al., "Secondary outcomes in a clinical trial of carotenoids with coantioxidants versus placebo in early age-related macular degeneration," Ophthalmology 120(3), 600-606 (2013).

29. K. Neelam et al., "Measurement of macular pigment: Raman spectroscopy versus heterochromatic flicker photometry," Invest. Ophthalmol. Visual Sci. 46(3), 1023-1032 (2005).

30. R. E. Hogg et al., "In vivo macular pigment measurements: a comparison of resonance Raman spectroscopy and heterochromatic flicker photometry," Br. J. Ophthalmol. 91(4), 485-490 (2007).

31. A. M. Pawlak et al., "Advanced glycation as a basis for understanding retinal aging and noninvasive risk prediction," Ann. N. Y. Acad. Sci. 1126, 59-65 (2008).
32. E. Cinotti et al., "Optical diagnosis of a metabolic disease: cystinosis," J. Biomed. Opt. 18(4), 046013 (2013).

33. Q. Wang et al., "Detection and characterization of glaucoma-like canine retinal tissues using Raman spectroscopy," J. Biomed. Opt. 18(6), 067008 (2013).

34. R Core Team, "R: a language and environment for statistical computing," 2017, http://www.R-project.org/.

35. C. Beleites and V. Sergo, "hyperSpec: a package to handle hyperspectral data sets in R," 2018, http://hyperspec.r-forge.r-project.org.

36. C. Beleites, "cbmodels: collection of 'combined' models: PCA-LDA, PLS-LDA, PLS-LR as well as EMSC," $\mathrm{R}$ package version 0.520150729 (2015).

37. O. Ryabchykov et al., "Automatization of spike correction in Raman spectra of biological samples," Chemom. Intell. Lab. Syst. 155, 1-6 (2016).

38. A. Bonifacio et al., "Chemical imaging of articular cartilage sections with Raman mapping, employing uni- and multi-variate methods for data analysis," Analyst 135(12), 3193-3204 (2010).

39. F. Murtagh and P. Legendre, "Ward's Hierarchical agglomerative clustering method: which algorithms implement ward's criterion?" J. Classif. 31(3), 274-295 (2014).

40. L. Ginner et al., "Wide-field OCT angiography at $400 \mathrm{KHz}$ utilizing spectral splitting," Photonics 1(4), 369-379 (2014).

Clara Stiebing studied physics at the Goethe University, Frankfurt/ Main, Germany, during which she spend a semester at the Stockholm University, Sweden. She received her PhD from the Friedrich-Schiller-University Jena, Germany, in 2017. Currently, she works as a postdoctoral researcher at the Leibniz-IPHT, Jena, Germany, within the Spectroscopy and Imaging Research Department of Prof. Jürgen Popp. Her interests include the research and development of Raman spectroscopic techniques for life science applications.

Iwan W. Schie studied medical engineering at Beuth University of Applied Science, Berlin, and received his $\mathrm{PhD}$ in biomedical engineering from the University of California, Davis. Since 2014, he has been working as a postdoctoral researcher at the Leibniz-IPHT, where he established the group for "Multimodal Instrumentation" in 2017, with the research focus on high-throughput Raman spectroscopy systems for single-cell classification, fiber-probe development, and instrumentation for medical in vivo applications.

Michael Schmitt received his $\mathrm{PhD}$ in chemistry from the University of Würzburg in 1998. From 1999 to 2000, he went for postgraduate studies to the Steacie Institute for Molecular Sciences at the National Research Council of Canada. Within the group of Prof. Kiefer at the University of Würzburg, he finished his habilitation in 2004. Since 2004 , he has been a research associate in the group of Prof. Jürgen Popp at the Institute for Physical Chemistry of Friedrich-SchillerUniversity Jena. His main research interests are focused on nonlinear spectroscopy.

Izabella J. Jahn studied technological physics in Cluj Napoca, Romania. She received her PhD from the Friedrich-Schiller-University Jena, Germany, in 2016. Her attention was focused on the combination of microfluidics with surface enhanced Raman spectroscopy for drug detection. Currently, she is a postdoc in the workgroup of Prof. Jürgen Popp and explores the potential of Raman spectroscopy for biomedical applications.

Martin Jahn studied technical physics at the TU IImenau and received his doctorate in 2017 from Friedrich-Schiller-University Jena on the detection and quantification of life science relevant substances by means of surface enhanced Raman spectroscopy. Since 2018, he has been a project leader for microoptical systems at CiS Forschungsinstitut für Mikrosensorik $\mathrm{GmbH}$.

Amanda J. Kiliaan and her research group focus on neuroimaging research on cerebral hemodynamics and gray/white matter integrity in (1) mouse models for neurovascular/neurodegenerative diseases (hypertension, stroke, obesity, and dementia) and nutritional impact thereon; (2) postmortem human brain vascular and Alzheimer pathology; (3) morbid obese patients after bariatric surgery, in collaboration with clinical research groups and (food) industries. She has written more than 114 publications, $\mathrm{H}$ index 36 . 
Antonia Lichtenegger received her master's degrees in technical mathematics and biomedical engineering from the Technical University of Vienna in 2014 and 2015, respectively. She is currently a PhD candidate at the Center for Medical Physics and Biomedical Engineering of the Medical University of Vienna. Her interests include designing and setting up optical coherence microscopy systems and image processing in biomedical optics and neuroscience.

Jürgen Popp studied chemistry at the Universities of Erlangen and Würzburg. After his $\mathrm{PhD}$ in chemistry, he joined Yale University for postdoctoral work. He returned to Würzburg where he finished his habilitation in 2002. Since 2002, he has been a chair for physical chemistry at Friedrich-Schiller-University Jena. Since 2006, he has been also the scientific director of the Leibniz-IPHT, Jena, Germany. His research interests are mainly concerned with biophotonics, in particular the development and application of innovative Raman techniques for biomedical diagnosis.

Biographies of the other authors are not available. 